\title{
Efficacy of bedaquiline in the treatment of drug-resistant tuberculosis: a systematic review and meta-analysis
}

\author{
Ming-Gui Wang, Shou-Quan Wu and Jian-Qing He*
}

\begin{abstract}
Background: Drug-resistant tuberculosis (DR-TB) remains a major public health concern worldwide. Bedaquiline, a novel diarylquinoline, was added to the WHO-recommended all-oral regimen for patients with multidrug-resistant tuberculosis. We performed a systematic review and meta-analysis to determine the effect of bedaquiline on tuberculosis treatment outcomes.

Methods: We searched the PubMed, Web of Science and EMBASE databases for relevant studies published up to March 12, 2021. We included studies in which some participants received bedaquiline and others did not. Stata version 16.0 (Stata Corp., College Station, Texas, USA) was used to analyze the results of the meta-analysis. Risk ratios (RRs) with 95\% confidence intervals (95\% Cls) were calculated to evaluate the effect of bedaquiline on drug-resistant tuberculosis. Between-study heterogeneity was examined by the I-squared test. Randomized controlled trials were assessed for quality using the Jadad scale, and cohort studies were assessed using the Newcastle-Ottawa scale.
\end{abstract}

Results: Eight studies, including 2 randomized controlled trials and 6 cohort studies involving a total of 21,836 subjects, were included. When compared with the control, bedaquiline treatment was associated with higher rates of culture conversion (risk ratio (RR):1.272 (1.165-1.389), $\mathrm{P}<0.001)$. We found substantial evidence of a significant reduction in all-cause death (RR: $0.529(0.454-0.616), P<0.001)$ ) in the bedaquiline treatment group. There was no significant reduction in treatment success ( $R R=0.980(0.948-1.013, P=0.234)$ ).

Conclusions: This study demonstrated that compared with patients who do not receive bedaquiline, this drug has the potential to achieve a higher culture conversion rate and a lower mortality risk among drug-resistant tuberculosis cases.

Keywords: Bedaquiline, Tuberculosis, Multidrug resistance, Extensively drug resistant

\section{Background}

Tuberculosis (TB) remains an important global infectious disease. TB is caused by mycobacterium tuberculosis (MTB) and remains one of the leading causes of infection-related death worldwide. According to the World Health Organization (WHO), there were 10.0 million

*Correspondence: Jianqhe@gmail.com; jianqing_he@scu.edu.cn Department of Respiratory and Critical Care Medicine, West China Hospital, Sichuan University, No. 37, Guo Xue Alley, Chengdu, Sichuan, People's Republic of China (range, 8.9-11.0 million) new TB patients in 2019 [1]. Globally, an estimated 1.4 million TB deaths occurred in 2019, including 1.2 million among human immunodeficiency virus (HIV)-negative people and an additional 208,000 deaths among HIV-positive people [1]. Drugresistant TB (DR-TB) is a major public health concern. Rifampicin-resistant TB (RR-TB) requires treatment with second-line drugs. Multidrug-resistant TB (MDR-TB) is resistant to both rifampicin and isoniazid (the two most effective anti-TB drugs), and extensively drug-resistant 
tuberculosis (XDR-TB) is MDR-TB that is also resistant to fluoroquinolone, and injectable agent. Globally, in $2019,3.3 \%$ of new cases and $18 \%$ of previously treated cases had MDR/RR-TB. It is estimated that there were 465000 incident cases of MDR/RR-TB in 2019, and the global proportion of RR-TB cases estimated to have MDR-TB was $78 \%$ [1]. The three countries with the heaviest burden of drug-resistant tuberculosis are India, China and the Russian Federation [1].

In 2019, total of $177,099 \mathrm{MDR} / \mathrm{RR}-\mathrm{TB}$ patients were reported to have received treatment [1], up from 156,205 in 2018 . However, $86 \%$ of the 206,030 people with MDR/ RR-TB who were detected and notified in 2019 started MDR-TB treatment. Treatment outcomes for MDR/ RR-TB remain poor even in advanced healthcare systems. Overall, only $57 \%$ of MDR/RR-TB patients in the 2017 cohort successfully completed treatment (cured or treatment completed) [1]. Hence, unsuccessful treatment of MDR-TB is a key problem that requires action.

The novel diarylquinoline, bedaquiline, was added to the WHO-recommended all-oral regimen to replace the injectable treatments for MDR-TB patients [2]. Bedaquiline has been shown to improve sputum conversion rates in clinical studies $[3,4]$ and was shown to improve treatment outcomes in some observational studies [5-7]. It is thus necessary to review and summarize the overall treatment outcomes for MDR-TB patients who were treated with bedaquiline in recent years. We conducted this systematic review and meta-analysis to summarize the existing evidence of the effect of bedaquiline on DR-TB treatment outcomes.

\section{Methods}

The meta-analysis was prepared based on the Preferred Reporting Items for Systematic Reviews and Meta-Analyses guidelines for systematic reviews and meta-analyses [8]. Since this was a meta-analysis of existing articles and no individual patient data were handled, ethical approval was unnecessary for this study.

\section{Search strategy and study selection}

The PubMed, Web of Science and EMBASE databases was searched to identify relevant studies (Additional file 1: Table S1). English-language studies published until March 12, 2021, were retrieved using the following keywords: "bedaquiline", or "tuberculosis," or "multidrug resistant tuberculosis" or "extensively drug resistant tuberculosis", and their synonyms or similar words. Two independent reviewers (MG and SQ) read and assessed the titles and abstracts of all articles identified by the search strategy. The full-text study reports of all potentially eligible studies were also independently screened by two review authors (MG and SQ) according to a standardized form containing the inclusion and exclusion criteria.

The inclusion criteria were: (1) patients were aged $\geq 18$ years; (2) had laboratory-confirmed DR-TB; (3) and received anti-TB therapy containing bedaquiline as an intervention; (4) the control group was treated with drugs other than bedaquiline; (5) culture conversion or outcomes of success (including cure or treatment completion), failure, and death according to the WHO classification were reported [9]; and (6) the study was designed as a retrospective study, randomized controlled trial, or prospective cohort study. When data were duplicated or reported in more than one study, the first published study was included in the meta-analysis.

Articles were excluded if they were editorials, case reports, conference abstracts, animal studies, or had a sample size of less than 10 .

\section{Assessment of methodological quality}

All studies included in the meta-analysis were independently assessed for quality by 2 reviewers, and the highquality studies were further analyzed. For randomized controlled trials (RCTs), the two review authors independently used the Jadad scale [10] to assess the methodological quality of each included study by using the following variables: random scheme and allocation concealment, blinding of participants, and follow-up. The maximum score was five points. A score of $\geq 3$ was considered to indicate high quality. For cohort studies, the quality of studies was assessed with a modified version of the Newcastle-Ottawa scale (NOS) (http://www.ohri. $\mathrm{ca} /$ programs/clinical_epidemiology/oxford.asp) by two reviewers independently. Studies were evaluated on the basis of adequate participant selection, comparability of studies based on design and analysis, and adequate ascertainment of outcomes. This scale awards a maximum of nine points. A score of $>7$ was considered to indicate high quality.

\section{Data extraction}

Two review authors (MG and SQ) worked independently to extract data on the following characteristics: study characteristics (author; publication year; country, study design), characteristics of participants (sample size, gender, age, HIV coinfection), intervention arms and controls (intervention drug and dose, follow-up duration, and anti-TB therapy protocol), and treatment outcomes (culture conversion, treatment success (cure or treatment completed), and death). Disagreements were resolved through discussion and consensus. 


\section{Statistical analysis}

All the statistical analyses were performed by using the Stata version 16.0 (Stata Corp., College Station, Texas, USA). To evaluate the effect of bedaquiline on drug-resistant tuberculosis, meta-analysis calculations were performed using individual data from patients with clear treatment outcomes (culture conversion and treatment success (including cure or treatment completion) and all-cause mortality). The risk ratio (RR) and 95\% confidence interval ( $95 \% \mathrm{CI})$ was used as the measure of treatment outcome (all-cause mortality, culture conversion, or treatment success). Between-study heterogeneity was examined by the I-squared test [11]. Publication bias was tested by Egger's linear regression test and Begg's test.

\section{Results}

Study flow diagram

A total of 3484 citations were identified from the scientific literature search. After duplicates were removed, the title and abstract of 2041 records were screened, and 80 articles were found to be relevant for full-text analysis and reference list screening. From these, 72 articles did not fulfil the inclusion criteria and were excluded, and 8 studies were identified as eligible for inclusion in the meta-analysis [3-7, 12-14] (Fig. 1).

\section{Characteristics of included studies}

The characteristics of the studies and the number of cases analyzed in the systematic review and meta-analysis are summarized in Table 1 . The 8 included studies were
3484 records identified through database searching

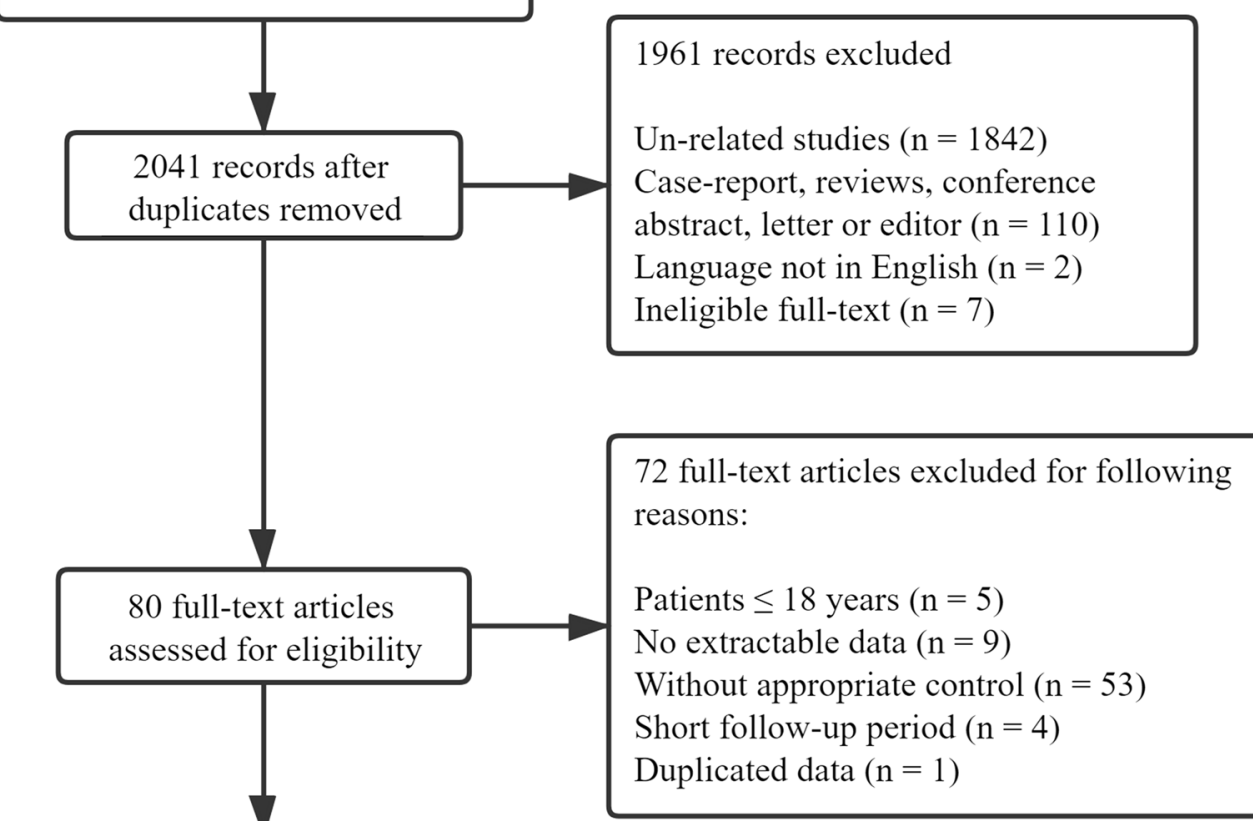

8 studies included in review

2 randomized; 3 prospective cohort; 3 retrospective cohort

8 studies eligible for meta-analysis

Fig. 1 Study flow diagram 


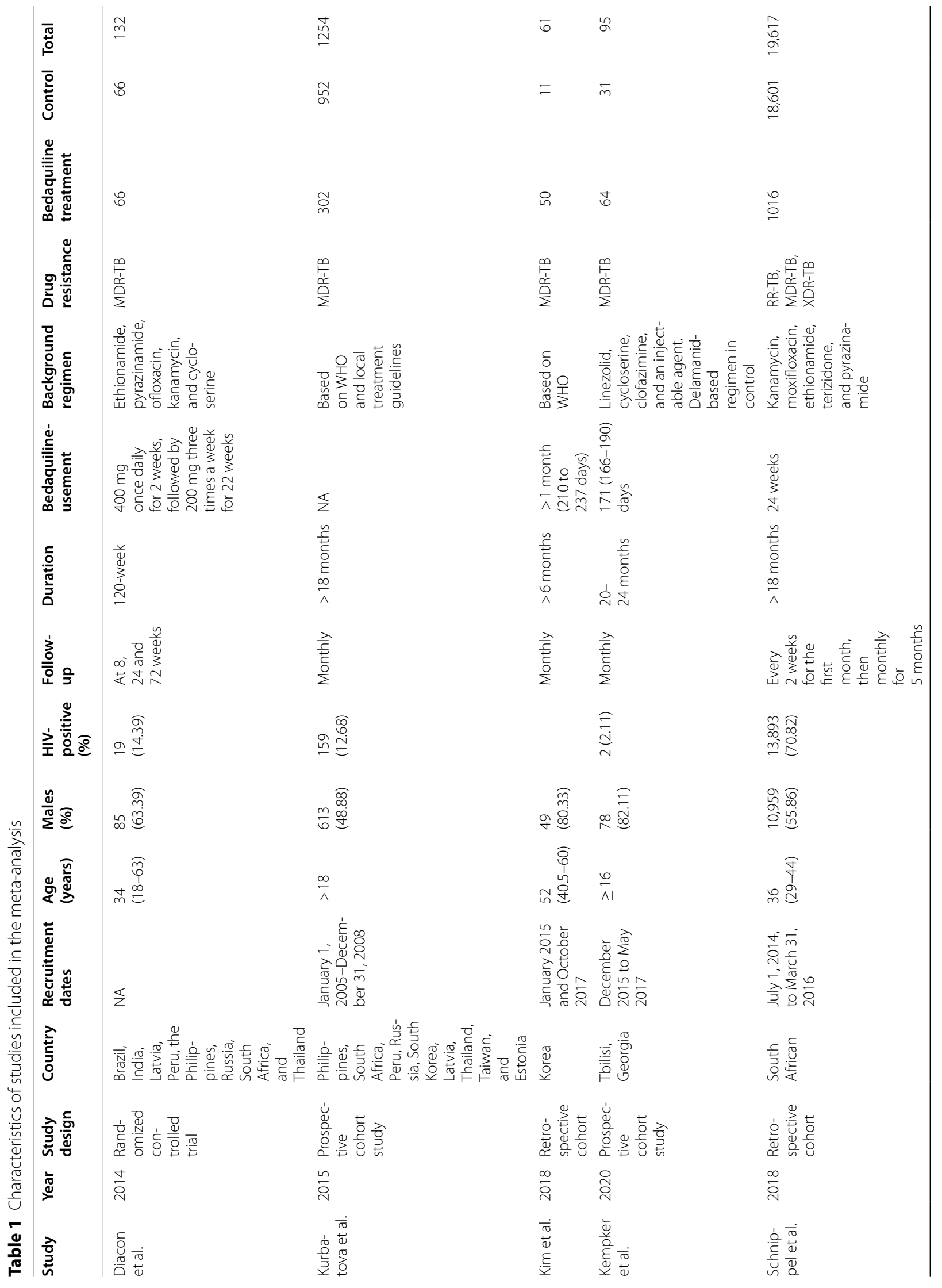




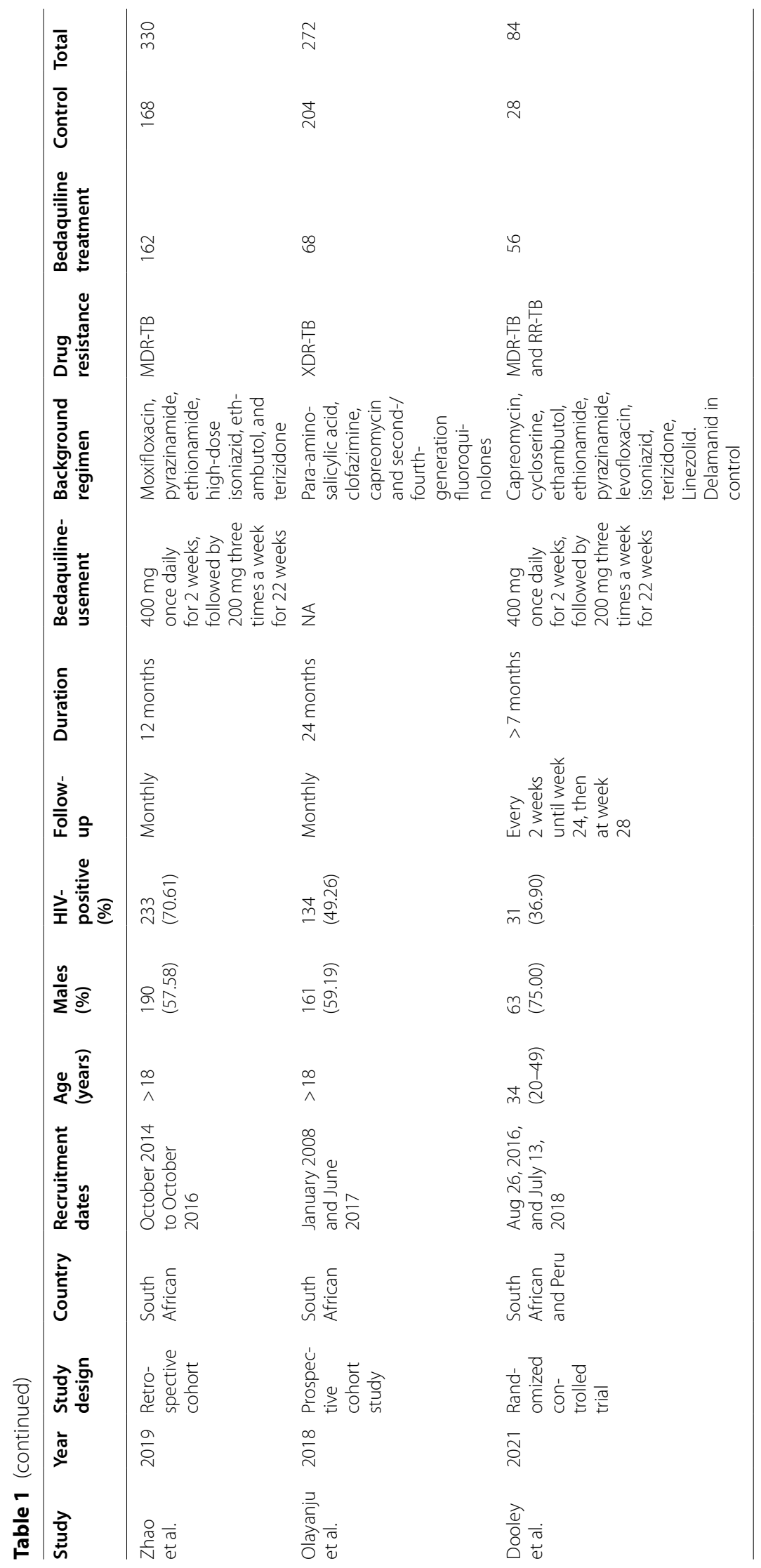


conducted in 13 countries. Regarding regional distribution, more than half were conducted in South Africa [3$7,14]$ (Table 1). Approximately $75 \%(n=6)$ of the studies were published in the last 5 years. Of the eight included studies, two were RCTs [3, 4], three were retrospective cohort studies, and three were prospective cohort studies $[5-7,12-14]$.

In total, there were 21,845 patients from the 8 included studies (Table 1), including 1784 patients treated with bedaquiline and 20,061 not treated with bedaquiline. Nearly $66.3 \%$ were HIV positive, and $55.9 \%$ were males. The antiviral treatment of HIV-positive patients in both the case group and the control group was consistent among the studies. In the bedaquiline treatment group, bedaquiline was generally administered at $400 \mathrm{mg}$ daily for 2 weeks, followed by $200 \mathrm{mg}$ three times per week for 22 weeks. The duration of treatment was $>6$ months. The sample sizes of the studies included in the meta-analysis ranged from 61 [12] to 19,617 [7].

\section{Treatment outcomes}

The meta-analysis found that the risk of culture conversion was higher in patients receiving bedaquiline-containing regimens than in those not receiving bedaquiline-containing regimens (RR: 1.272 (1.1651.389), $\mathrm{P}<0.001$ ) (Fig. 2). However, bedaquiline treatment did not have a statistically significant effect on the outcome of success (RR: $0.980(0.948-1.013), \mathrm{P}=0.234$ )
(Fig. 3). There were significant differences in the proportion of deaths due to any cause between those who received bedaquiline-containing regimens versus the controls. Patients receiving bedaquiline had a lower risk of all-cause mortality than those not receiving bedaquiline (RR: 0.529 (0.454-0.616), $\mathrm{P}<0.001$ ) (Fig. 4).

Significant heterogeneity was detected between the results of the studies, with an $\mathrm{I}^{2}$ value of $91.4 \%$ for culture conversion, $94.8 \%$ for successful treatment, and $62.6 \%$ for all-cause mortality. Due to the significant heterogeneity, we performed sensitivity analyses to explore the sources of heterogeneity. The heterogeneity was significantly reduced after the removal of Diacon (2014) (from 62.6\% to $6.2 \%$ ) for all-cause mortality [4].

\section{Assessment of risk of bias and publication bias}

We assessed the risk of bias for the included RCTs using the Jadad scale, and the two included RCTs were of high quality (score $\geq 3$ ). For cohort studies, we assessed the risk of bias using the NOS tool, and all included cohort studies were considered to be high quality. The results of the risk of bias analysis for the included studies are summarized in Additional file 1: Tables S2 and S3.

Begg's and Egger's regression tests were performed to assess publication bias. No substantial publication bias was found by either test. The Begg's funnel plot is shown in Fig. 5.

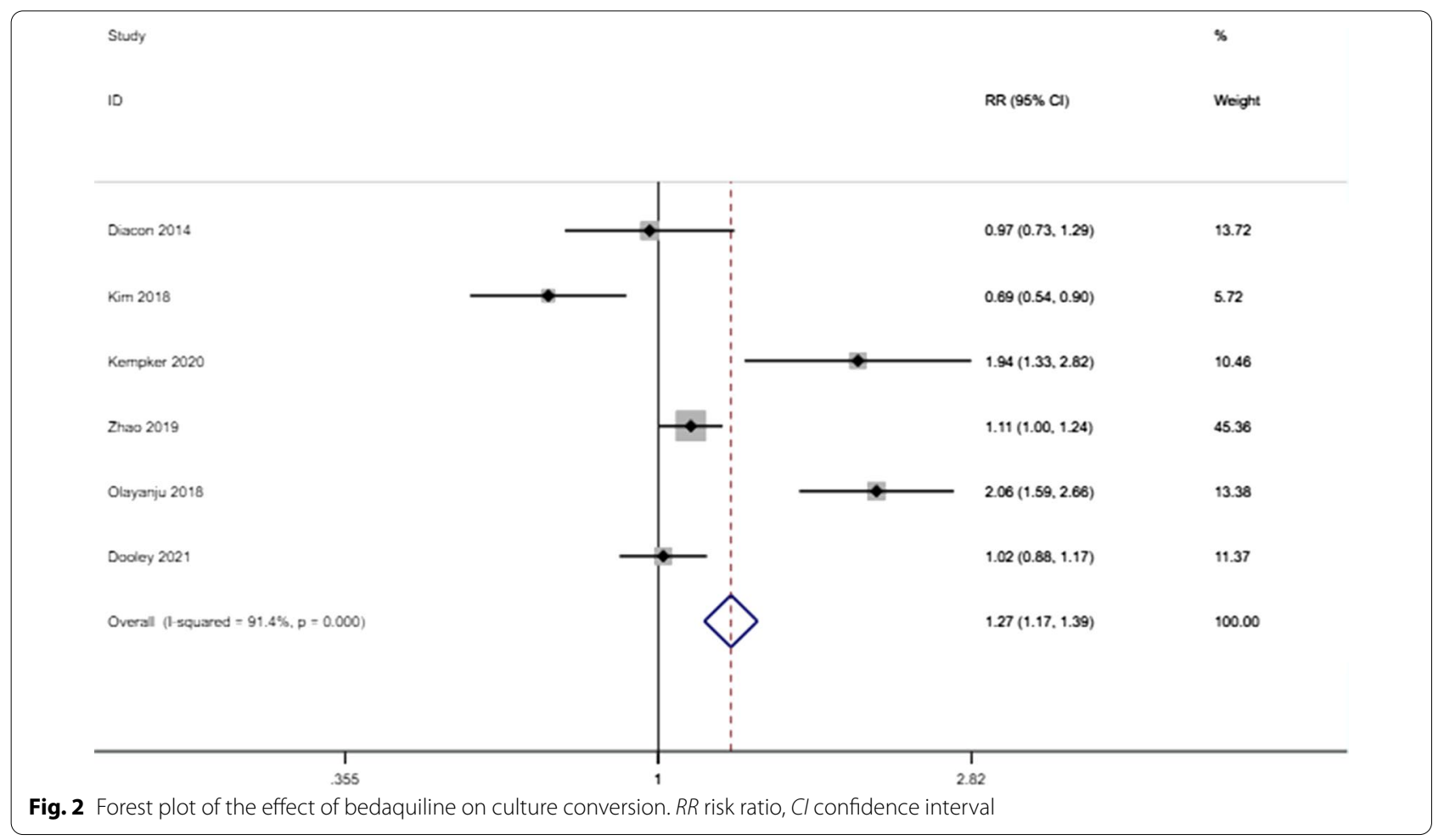


Study

10

Discon 2014

Kurbatova 2015

Kin 2018

Kempker 2020

Schnippel 2018

Zhao 2019

Oayanju 2018

Overall (1-squared $=94.8 \%, p=0.000$ )

267

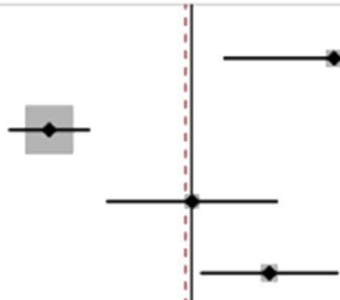

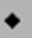

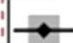

RR ( $95 \%$ CI)

$\%$

Weight

1.60

22.13

$1.00(0.74,136)$

$1.32(1.03,1.69)$

$1.02(1.00,1.05) \quad 64.43$

$1.14(1.02,1.27)$

$\longrightarrow 2.72(1.98,3.74)$

$0.98(0.95,1.01) \quad 100.00$

Fig. 3 Forest plot of the effect of bedaquiline on treatment success. $R R$ risk ratio, $C /$ confidence interval

\section{Study}

10

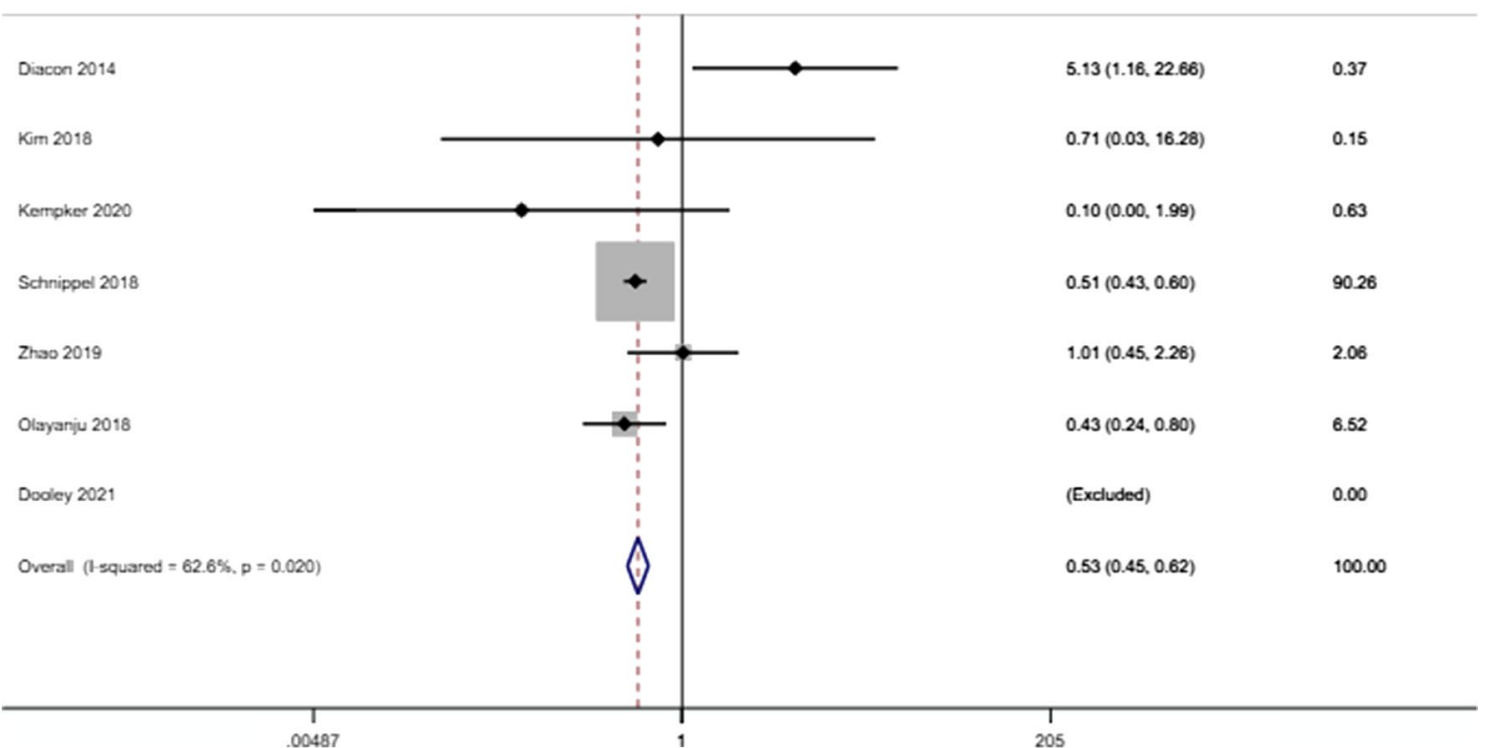

Fig. 4 Forest plot of the effect of bedaquiline on all-cause mortality. $R R$ risk ratio, Cl confidence interval 


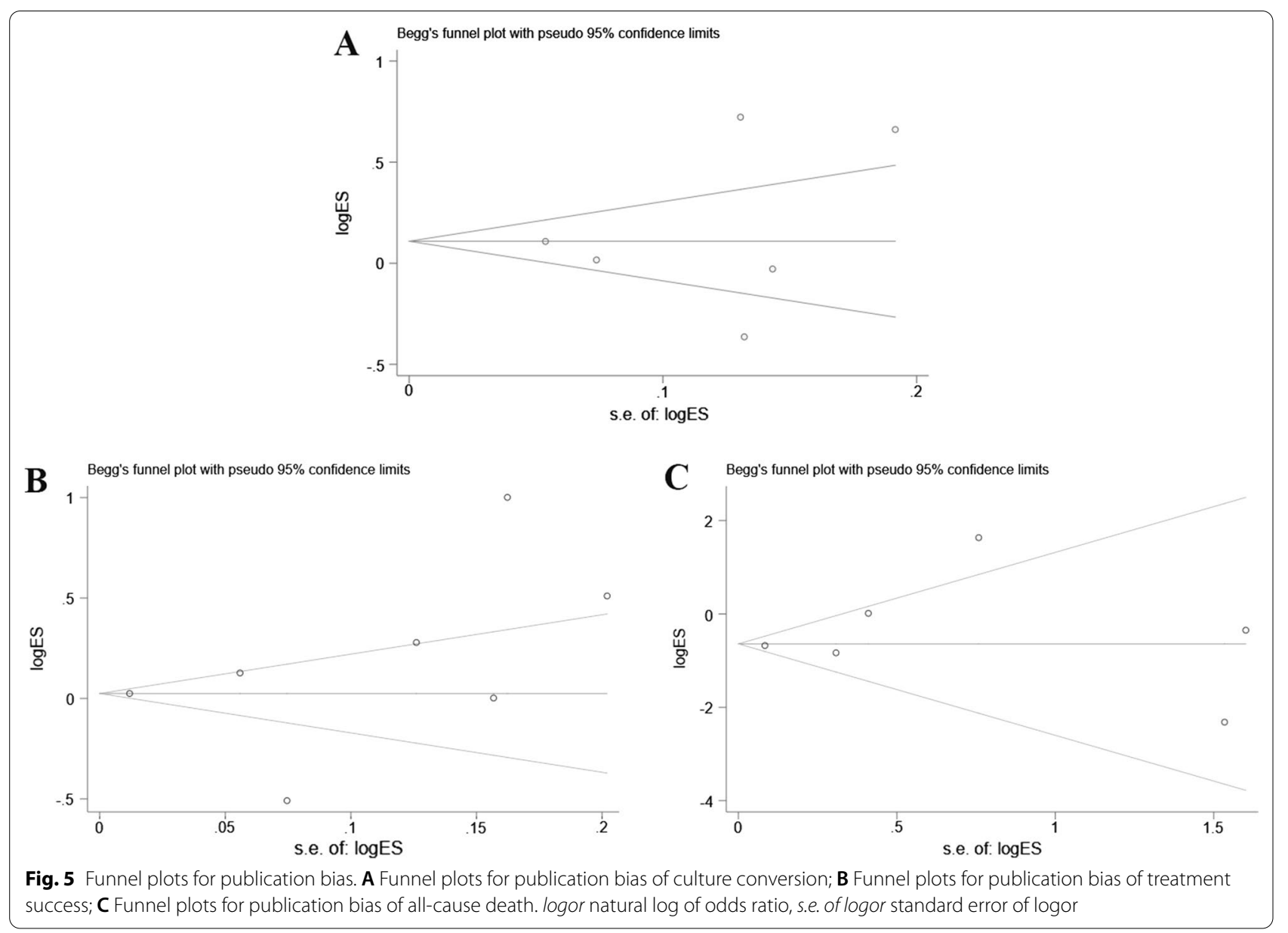

\section{Discussion}

To our knowledge, this is the first meta-analysis to investigate the effects of bedaquiline on patients with DR-TB. We analyzed data from 8 studies conducted in 13 countries, including 21,836 DR-TB patients. The results of this meta-analysis revealed the efficacy of bedaquiline in the treatment of drug-resistant tuberculosis.

For DR-TB, especially MDR/RR-TB and XDR-TB, bedaquiline was always administered in combination with other antitubercular drugs. Thus, treatment outcomes may not be entirely attributable to bedaquiline. Nevertheless, since all patients with DR-TB were treated with a background regimen, we believe that bedaquiline may be the most important factor affecting the treatment outcome in this meta-analysis. We found that bedaquiline could increase culture conversion (RR: 1.272 (1.1651.389), $\mathrm{P}<0.001)$ and decrease the risk of all-cause mortality (RR: 0.529 (0.454-0.616), $\mathrm{P}<0.001)$. However, the administration of bedaquiline did not increase treatment success among DR-TB patients $(\mathrm{P}=0.234)$.

Bedaquiline is a new antituberculosis drug belonging to the diarylquinoline class of compounds. It contains a quinolinic central heterocyclic nucleus with alcohol and amine side chains that play an important role in antituberculosis activity [15]. Studies have shown that bedaquiline is an inhibitor of mycobacterial ATP synthase; it binds to and perturbs the a-c subunit interface of Fo and leads to an ineffective proton cycle, which is fatal to mycobacterium $[16,17]$. A multicenter study conducted in 25 centers and 15 countries on five continents found that at the end of treatment, the negative sputum smear and culture conversion rates in MDR-TB cases were $88.7 \%$ and $91.2 \%$, respectively, and $71.3 \%$ achieved treatment success [18]. In other words, bedaquiline-containing regimens achieved high conversion and success rates when used to treat MDR-TB patients [18]. Another retrospective French cohort study showed that $97 \%$ of culture-positive TB patients achieved culture conversion after 6 months of bedaquiline treatment [19]. Our study evaluated the efficacy of bedaquiline for the treatment of DR-TB in RCTs and cohort studies. We found that DR-TB patients can benefit from the use of bedaquiline; such treatment can achieve a better sputum conversion rate and a lower risk of death. The WHO consolidated 
guidelines on DR-TB treatment recommend bedaquiline as one of the priority drugs (group A) for MDR-TB patients [2]. The use of bedaquiline may constitute a new era in the treatment of DR-TB patients, contributing to curbing the spread of this disease and reducing its mortality.

Taune et al. conducted a retrospective cohort study to describe the implementation of bedaquiline treatment and assess the safety and interim effectiveness for MDRTB patients commenced on bedaquiline. The results showed that bedaquiline is a safe and well-tolerated drug with good interim effectiveness [20]. Studies of children and adolescents with DR-TB also show that bedaquilinecontaining regimens are effective and well tolerated in children and adolescents, which may provide new directions for tuberculosis treatment in this group and contribute to the global strategy to end tuberculosis [20-22]. However, we did not evaluate the efficacy of bedaquiline in the treatment of child and adolescent patients with DR-TB, and further studies are needed.

There are many adverse reactions to bedaquiline, such as hyperuricemia, nausea, arthralgia, liver injury and QT prolongation [18-24]. Guglielmetti et al. found that nearly $20 \%$ of patients experienced a $>=60-\mathrm{ms}$ increase in QT interval, leading to bedaquiline discontinuation in $6 \%$ of patients [19]. A multicenter study found that adverse events presumably due to bedaquiline occurred in $19.4 \%$ of treated patients, and $5.8 \%$ of patients interrupted their bedaquiline treatment because of adverse events [18]. It is thought that most patients treated with bedaquiline will have adverse drug reactions, but most reactions are mild and do not lead to discontinuance [18, 19, 23, 25]. However, fatal arrhythmias can cause death [23].

Our review has some limitations. First, we included cohort studies and RCTs, which may have led to heterogeneity. Second, due to the limited data, we were unable to evaluate the safety of bedaquiline in the treatment of MDR/RR-TB and XDR-TB, and further studies are needed. In addition, we did not evaluate the effect of bedaquiline on DR-TB treatment outcomes among HIVpositive, child or adolescent patients. Further research focusing on these populations is necessary. Third, only eight studies were included in this meta-analysis, and the sample size of some of these studies was small. Additional randomized controlled trials with larger sample sizes are needed to further evaluate the efficacy and safety of bedaquiline in the treatment of DR-TB. Finally, our review processes had some limitations. To ensure feasibility, we were only able to include published articles, and unpublished articles were not screened. Furthermore, the language was limited to English, and articles published in other languages were not reviewed.

\section{Conclusion}

The use of bedaquiline combined with other active drugs has the potential to achieve a higher culture conversion rate and a lower mortality risk among MDR/RR-TB and XDR-TB patients compared with those who do not receive this drug. Thus, the use of bedaquiline in DR-TB patients should be encouraged.

\section{Abbreviations \\ TB: Tuberculosis; MTB: Mycobacterium tuberculosis; WHO: World Health Organization; HIV: Human immunodeficiency virus; DR-TB: Drug-resistant tuberculosis; RR-TB: Rifampicin-resistant TB; MDR-TB: Multidrug-resistant TB; XDR-TB: Extensively drug-resistant tuberculosis; RCT: Randomized controlled trial; NOS: Newcastle-Ottawa Assessment Scale; RR: Risk ratio; Cl: Confidence interval.}

\section{Supplementary Information}

The online version contains supplementary material available at https://doi. org/10.1186/s12879-021-06666-8.

Additional file 1: Table S1. Search strategy. Table S2. The Jadad scale of randomized controlled trials. Table S3. The Newcastle-Ottawa quality assessment scale of cohort studies.

\section{Acknowledgements}

Not applicable.

\section{Authors' contributions}

All authors contributed substantially to the study design, data interpretation, and the writing of the manuscript. Dr. JQH contributed to the study design. MGW and SQW contributed to data collection, completed full text. All authors reviewed, read and approved the final manuscript.

\section{Funding}

This work was supported by the National Natural Science Foundation of China (Grant No. 81870015), which contributed to the study design.

\section{Availability of data and materials}

The datasets used and/or analysed during the current study are available from the corresponding author on reasonable request.

\section{Declarations}

Ethics approval and consent to participate Not applicable.

\section{Consent for publication}

Not applicable.

\section{Competing interests}

The authors declare that they have no competing interests.

Received: 12 May 2021 Accepted: 7 September 2021

Published online: 17 September 2021

References

1. Global tuberculosis report 2020. Geneva: World Health Organization; 2020. Licence: CC BY-NC-SA 3.0 IGO.

2. WHO consolidated guidelines on drug-resistant tuberculosis treatment. Geneva: World Health Organization; 2019. Licence: CC BY-NC-SA 3.0 IGO.

3. Dooley KE, Rosenkranz SL, Conradie F, Moran L, Hafner R, von GrooteBidlingmaier F, Lama JR, Shenje J, De Los Rios J, Comins K, et al. QT effects 
of bedaquiline, delamanid, or both in patients with rifampicin-resistant tuberculosis: a phase 2, open-label, randomised, controlled trial. Lancet Infect Dis. 2021;21(7):975-83.

4. Diacon AH, Pym A, Grobusch MP, de los Rios JM, Gotuzzo E, Vasilyeva I, Leimane V, Andries K, Bakare N, De Marez T, et al. Multidrug-resistant tuberculosis and culture conversion with bedaquiline. $\mathrm{N}$ Engl I Med. 2014;371(8):723-32

5. Olatunde O, Jason L, Aliasgar E, Suzette O, Phindile G, Elize P, Mohammed F, Rob W, Keertan D. Long-term bedaquiline-related treatment outcomes in patients with extensively drug-resistant tuberculosis from South Africa. Eur Respir J. 2018;51(5):1800544.

6. Kurbatova EV, Dalton T, Ershova J, Tupasi T, Caoili JC, Walt MVD, Kvasnovsky C, Yagui M, Bayona J, Contreras C, et al. Additional drug resistance of multidrug-resistant tuberculosis in patients in 9 countries. Emerg Infect Dis. 2015;21 (6):977-83.

7. Schnippel K, Ndjeka N, Maartens G, Meintjes G, Master I, Ismail N, Hughes $J$, Ferreira $H$, Padanilam X, Romero R, et al. Effect of bedaquiline on mortality in South African patients with drug-resistant tuberculosis: a retrospective cohort study. Lancet Respir Med. 2018;6(9):699-706.

8. Moher D, Liberati A, Tetzlaff J, Altman DG, Group P. Preferred reporting items for systematic reviews and meta-analyses: the PRISMA statement. Ann Intern Med. 2009;151(4):264-269264.

9. In: Guidelines for the programmatic management of drug-resistant tuberculosis: 2011 update Geneva: WHO; 2011. edn. Geneva.

10. Jadad AR, Moore RA, Carroll D, Jenkinson C, Reynolds DJ, Gavaghan DJ, McQuay HJ. Assessing the quality of reports of randomized clinical trials: is blinding necessary? Control Clin Trials. 1996;17(1):1-12.

11. Higgins JP, Thompson SG, Deeks JJ, Altman DG. Measuring inconsistency in meta-analyses. BMJ. 2003;327(7414):557-60

12. Kim CT, Kim T-O, Shin H-J, Ko YC, Choe YH, Kim H-R, Kwon Y-S. Bedaquiline and delamanid for the treatment of multidrug-resistant tuberculosis: a multicentre cohort study in Korea. Eur Respir J. 2018. https://doi.org/10. 1183/13993003.02467-2017.

13. Kempker RR, Mikiashvili L, Zhao Y, Benkeser D, Barbakadze K, Bablishvili N, Avaliani Z, Peloquin CA, Blumberg HM, Kipiani M. Clinical outcomes among patients with drug-resistant tuberculosis receiving bedaquilineor delamanid-containing regimens. Clin Infect Dis. 2020;71(9):2336-44.

14. Zhao Y, Fox T, Manning K, Stewart A, Tiffin N, Khomo N, Leslie J, Boulle A, Mudaly V, Kock Y, et al. Improved treatment outcomes with bedaquiline when substituted for second-line injectable agents in multidrugresistant tuberculosis: a retrospective cohort study. Clin Infect Dis. 2019:68(9):1522-9.

15. Matteelli A, Carvalho AC, Dooley KE, Kritski A. TMC207: the first compound of a new class of potent anti-tuberculosis drugs. Future Microbiol. 2010;5(6):849-58.
16. Hards K, Robson JR, Berney M, Shaw L, Bald D, Koul A, Andries K, Cook GM. Bactericidal mode of action of bedaquiline. J Antimicrob Chemother. 2015;70(7):2028-37.

17. Koul A, Dendouga N, Vergauwen K, Molenberghs B, Vranckx L, Willebrords R, Ristic Z, Lill H, Dorange I, Guillemont J, et al. Diarylquinolines target subunit c of mycobacterial ATP synthase. Nat Chem Biol. 2007;3(6):323-4.

18. Borisov SE, Dheda K, Enwerem M, Leyet RR, D'Ambrosio L, Centis R, Sotgiu G, Tiberi S, Alffenaar J-W, Maryandyshev A, et al. Effectiveness and safety of bedaquiline-containing regimens in the treatment of MDR- and XDRTB: a multicentre study. Eur Respir J. 2017;49(5):1700387.

19. Guglielmetti L, Le Du D, Jachym M, Henry B, Martin D, Caumes E, Veziris N, Metivier N, Robert J. French MDRTBC: compassionate use of bedaquiline for the treatment of multidrug-resistant and extensively drugresistant tuberculosis: interim analysis of a French cohort. Clin Infect Dis. 2015;60(2):188-94.

20. Taune M, Ustero P, Hiashiri S, Huang K, Aia P, Morris L, Main S, Chan G, du Cros P, Majumdar SS. Successful implementation of bedaquiline for multidrug-resistant TB treatment in remote Papua New Guinea. Public Health Action. 2019;9:573-9.

21. Solodovnikova V, Kumar AM, Hurevich H, Sereda Y, Auchynka V, Katovich D, Klimuk D, Skrahin A, Setkina S, Charnysh I, et al. Effectiveness and safety of delamanid-or bedaquiline-containing regimens among children and adolescents with multidrug resistant or extensively drug resistant tuberculosis: a nationwide study from Belarus, 2015-19. Monaldi Arch Chest Dis. 2021. https://doi.org/10.4081/monaldi.2021.1646.

22. Das M, Mamnoon F, Mansoor H, Meneguim AC, Singh P, Shah I, Ravi S, Kalon S, Hossain FN, Ferlazzo G, et al. NewTB drugs for the treatment of children and adolescents with rifampicin-resistant TB in Mumbai, India. Int J Tuberc Lung Dis. 2020;24(12):1265-71.

23. Jones J, Mudaly V, Voget J, Naledi T, Maartens G, Cohen K. Adverse drug reactions in South African patients receiving bedaquiline-containing tuberculosis treatment: an evaluation of spontaneously reported cases. BMC Infect Dis. 2019;19(1):544.

24. Pym AS, Diacon AH, Tang SJ, Conradie F, Danilovits M, Chuchottaworn C, Vasilyeva I, Andries K, Bakare N, De Marez T, et al. Bedaquiline in the treatment of multidrug- and extensively drug-resistant tuberculosis. Eur Respir J. 2016;47(2):564-74.

25. Gaida R, Truter I, Peters CA. Adverse effects of bedaquiline in patients with extensively drug-resistant tuberculosis. S Afr J Infect Dis. 2020;35(1):1-6.

\section{Publisher's Note}

Springer Nature remains neutral with regard to jurisdictional claims in published maps and institutional affiliations.
Ready to submit your research? Choose BMC and benefit from:

- fast, convenient online submission

- thorough peer review by experienced researchers in your field

- rapid publication on acceptance

- support for research data, including large and complex data types

- gold Open Access which fosters wider collaboration and increased citations

- maximum visibility for your research: over 100M website views per year

At BMC, research is always in progress.

Learn more biomedcentral.com/submissions 